\title{
PANORAMA DOS PRINCIPAIS ESTUDOS SOBRE CIÊNCIA CIDADÃ ${ }^{1}$
}

\author{
Diny Gabrielly de Miranda Martins ${ }^{2}$ \\ Eloisa Helena de Souza Cabral
}

\begin{abstract}
RESUMO
Neste artigo, são analisados os objetos de estudo das principais pesquisas sobre ciência cidadã publicadas nos últimos cinco anos. Realizou-se uma busca na base de dados internacional Web of Science por artigos científicos publicados nos anos de 2016 a 2020 e com um número mínimo de 40 citações. Foram encontrados 31 artigos científicos internacionais que tratam de conceitos teóricos, aplicações e contribuições da Ciência Cidadã, abordam implicações éticas e sociais no recrutamento dos voluntários e a motivação desses cidadãos não cientistas para participarem de projetos de Ciência Cidadã e, ainda, avaliam a qualidade dos dados coletados. A pesquisa realizada pode contribuir para a academia, servir de base teórica para o tema da Ciência Cidadã e ser motivante para que os docentes conheçam e se utilizem de iniciativas nos diversos campos do conhecimento como abordagem no processo de ensino e aprendizagem.
\end{abstract}

Palavras-chave: Ciência cidadã. Participação. Não cientistas.

\section{OVERVIEW OF MAIN STUDIES ON CITIZEN SCIENCE}

\begin{abstract}
This article analyzes the objects of study of the main researches on citizen science published in the last five years. A search was made in the international Web of Science database for scientific articles published in the years 2016 to 2020 and with a minimum number of 40 citations. Thirtyone international scientific articles were obtained that deal with the theoretical concepts, applications and contributions of citizen science. They also address the ethical and social implications of recruiting volunteers and the motivation of these non-scientist citizens to participate in citizen science projects, and also assess the quality of the data collected. The research carried out can contribute to the academy, serving as a theoretical basis on the theme of citizen science and be motivating for teachers to know and use citizen science initiatives in different fields of knowledge as an approach in the teaching-learning process.
\end{abstract}

Keywords: Citizen Science. Participation. Non-scientist.

\section{INTRODUÇÃO}

A curiosidade, tomada como atributo humano, é característica de todas as pessoas e em relação à variedade dos problemas estabelecidos como fatos científicos, o interesse e a participação das pessoas, o espírito público e a divulgação dos achados científicos, podem

\footnotetext{
${ }^{1}$ MARTINS, D. G. M.; CABRAL, E. H. S. Panorama dos principais estudos sobre ciência cidadã. ForScience, Formiga, v. 9, n. 2, e01030, jul./dez. 2021. DOI: 10.29069/forscience.2021v9n2.e1030.
}

\section{${ }^{2}$ Autor para correspondência: Diny Gabrielly de Miranda Martins, e-mail: dinygabrielly@ hotmail.com.}


contribuir para a abordagem, o desenvolvimento e a utilização da Ciência como ferramenta para o progresso da sociedade humana.

Assim, despertar o interesse da sociedade civil pela Ciência e levar as pessoas a se envolverem com a pesquisa científica é fundamental para ampliar a construção do conhecimento e garantir avanços nas mais diversas áreas. A proposta pode ser colocada em prática por meio da chamada Ciência Cidadã, que se baseia na participação das pessoas em pesquisas e atividades científicas.

O termo, conhecido em inglês como Citizen Science, refere-se às parcerias entre cientistas e voluntários leigos, que atuam de forma efetiva em coletas e/ou análises de dados científicos relacionados às pesquisas aplicadas a temas de interesse público (DICKINSON ZUCKERBERG; BONTER, 2010). Também é empregado para descrever, de forma geral, projetos com finalidades científicas em que não cientistas cooperam como voluntários (SOARES; SANTOS, 2011).

Iniciativas de Ciência Cidadã podem ser utilizadas na Educação, como mecanismos que viabilizem o envolvimento da comunidade escolar com problemas reais da sociedade e levem os alunos à aprendizagem significativa, quando novos argumentos podem ser assimilados, respaldando ideias, conceitos e conhecimentos anteriores, num movimento no qual o que é integrado torna-se a substância do novo conhecimento. Nesse diálogo, o conhecimento precedente transforma-se, devido à obtenção de novas acepções. Ausubel (1963, p. 58) afirma que "a aprendizagem significativa é o mecanismo humano, por excelência, para adquirir e armazenar a vasta quantidade de ideias e informações representadas em qualquer campo de conhecimento".

Assim, a Ciência Cidadã e a aprendizagem significativa podem proporcionar oportunidades para que professores e alunos se aproximem da comunidade extraescolar e se envolvam com as questões sociais locais. Uma experiência baseada em questões locais, regionais e globais pode levar a mudanças mensuráveis na ação individual e na coletiva, principalmente por meio da participação em projetos de Ciência Cidadã e na ação e comunicação da comunidade (HAYWOOD; PARRISH; DOLLIVER, 2016).

A atuação em projetos fundamentados nos princípios da Ciência Cidadã faz com os participantes desempenhem uma atividade de aprendizado, ou lazer, por meio da qual colaboram formalmente com um projeto de pesquisa cujo resultado pode retornar para o participante e para a sociedade na forma de melhoria das condições sociais, ambientais ou mesmo na formulação de novas políticas públicas. 
Atualmente, uma variedade de projetos fundamentados na Ciência Cidadã, que perpassam pelas diferentes áreas de atuação científica e campos de conhecimento, tem sido praticada e estudada em todo o mundo. Entretanto, esse campo ainda é pouco divulgado e incentivado no Brasil, o que proporciona oportunidades de pesquisas inéditas sobre o tema.

Diante desse contexto, neste artigo, objetiva-se identificar quais são os objetos de estudo das principais pesquisas sobre Ciência Cidadã publicadas nos últimos cinco anos (2016-2020). Este estudo poderá servir como base teórica sobre o tema para a academia e ser motivante para que os docentes do Ensino Básico, Técnico ou Superior conheçam e se utilizem de iniciativas de Ciência Cidadã nos diversos campos do conhecimento como abordagem no processo de ensino e aprendizagem.

\section{REFERENCIAL TEÓRICO}

\subsection{Ciência Cidadã}

As pesquisas e o monitoramento de base comunitária vêm ganhando cada vez mais espaço, devido à importância dos resultados relativos à biodiversidade e preservação do meio ambiente. Estudos desse aspecto caracterizam-se pela interação de pesquisadores com o público leigo. Nesse contexto de participação do cidadão não cientista em pesquisas científicas é que o conceito de "ciência cidadã" ou "ciência aberta" costuma ser empregado. Dickinson, Zuckerberg e Bonter (2010), assim como Soares e Santos (2011), reforçam a importância das parcerias entre cientistas e voluntários leigos, os quais possuem efetiva participação em coletas e/ou análises de dados científicos relacionados às pesquisas aplicadas a temas de interesse público.

O conceito não é recente, pois práticas de Ciência Cidadã ocorrem há mais de um século, ainda que o termo não tivesse sido concebido na época. Segundo Rebouças (2013), a Ciência Cidadã tem origem entre o final do século XIX e início do século XX, mais precisamente, a partir de 1900, por meio da contagem coletiva de pássaros.

Trata-se de uma perspectiva metodológica para a produção do conhecimento, que se mostra como uma abordagem de pesquisa promissora para ser aplicada em diversos campos científicos. Essa abordagem destaca-se especialmente quanto ao seu potencial de geração de dados e análises, temporal e espacial, quando comparada à abordagem científica tradicional (PALMA, 2016).

O analista de política científica, Alan Irwin e, o ornitólogo e organizador da pesquisa participativa, Richard Boney, são frequentemente reconhecidos pela cunhagem do termo 
“ciência do cidadão" (IRWIN, 1995; BONNEY, 1996). Irwin (1995) define Ciência Cidadã como uma abordagem que atende às necessidades e preocupações dos cidadãos e, ao mesmo tempo, é desenvolvida e realizada pelos próprios cidadãos (IRWIN, 1995). Essa concepção é amplamente adotada nas Ciências Sociais e pelos formuladores de políticas que consideram os cidadãos como partes interessadas nos processos de tomada de decisão cientificamente informada (KULLENBERG; KASPEROWSKI, 2016).

Bonney (1996) define Ciência do Cidadão como projetos científicos nos quais os "amadores" fornecem dados observacionais como, por exemplo, de pássaros, para os cientistas e, adquirem novas habilidades científicas em troca. $\mathrm{O}$ autor entende a Ciência Cidadã como a participação do público na pesquisa científica e também como uma ferramenta para promover a compreensão pública da ciência (BONNEY, 1996).

O grupo de especialistas Socientize, da Unidade de Ciência Digital da Comissão Europeia, apresenta definição semelhante. Segundo ele, "ciência do cidadão refere-se ao envolvimento do público em geral em atividades de pesquisa científica quando os cidadãos contribuem ativamente para a ciência, seja com seu esforço intelectual, por meio da observação ou com suas ferramentas e recursos" (SOCIENTIZE, 2014, p. 1).

Para Cohn (2008), a Ciência Cidadã diz respeito a voluntários que participam como assistentes de campo em estudos científicos, ajudando no monitoramento de animais e plantas e/ou outros marcadores ambientais, mas não são remunerados e nem, necessariamente, cientistas. Na maioria, são amadores que se voluntariam para ajudar em pesquisas ecológicas, motivados por se sentirem bem ao ar livre, ou se preocuparem com os problemas ambientais, portanto, dispostos a contribuir para sanar essas problemáticas (COHN, 2008).

Segundo Cappa et al. (2016), a Ciência Cidadã é um meio de envolver o público em geral em atividades de pesquisa lideradas por cientistas profissionais. Ao envolver um grande número de não cientistas, a Ciência Cidadã permite a coleta e análise de dados distribuídos em uma escala que seria difícil obter, sobretudo, quando se consideram os recursos financeiros requeridos por determinados projetos.

Existem alguns sinônimos importantes para o conceito de Ciência Cidadã como "monitoramento baseado na comunidade", "monitoramento voluntário", "ciência participativa" e "ciência aberta", todos designando a contribuição de não cientistas para a ciência (KULLENBERG; KASPEROWSKI, 2016; CLÍNIO, 2019).

Ciência Cidadã é, portanto, uma forma humanística e colaborativa de conduzir estudos científicos, quando pesquisadores podem engajar muitas pessoas em suas pesquisas. Salientase que as pessoas não são apenas informadas sobre as descobertas científicas, mas participam 
do processo e são treinadas mediante o estabelecimento de protocolos específicos para cada projeto científico (BERTIN, 2019; JASANOFF, 2019).

Essa abordagem metodológica é de grande relevância para a Ciência, pois as colaborações entre cientistas e voluntários têm o potencial de ampliar o escopo da pesquisa e aprimorar a capacidade de coletar dados científicos. Membros do público interessados podem contribuir com informações valiosas, à medida que aprendem sobre questões relacionadas ao meio ambiente; à biomedicina; zoologia; biologia; às ciências sociais, com aplicabilidade tanto nas comunidades locais como nacionais (WILDSCHUT, 2017).

Um exemplo específico do campo das Matemáticas, para a qual a contribuição do Brasil é relevante, são os estudos da Etnomatemática, que é um esforço na fronteira da Antropologia Cultural, História da Ciência e da Heurística, com objetos matemáticos, principalmente os geométricos. Constitui-se, inicialmente, em um esforço de investigação histórico e cultural, para produzir resultados voltados ao entendimento e à comunicação de construções matemáticas; métodos de cálculos; símbolos; estruturas algébricas; e objetos, como desenhos gráficos e rede (D’AMBRÓSIO, 2001).

Domite (2005) apontou que o reconhecimento da origem de símbolos e métodos espontâneos do ato de contar pode ser absorvido e aplicado nos contextos cultural e escolar. Stewart (2005) indica que a arte de dobraduras de papel permite mostrar nivelamento de um sólido geométrico. Eglash (2005) identificou estruturas fractais em construções e urbanização de aldeias africanas.

Esses exemplos expandem a participação dos indivíduos e denotam que essas populações construíram objetos e símbolos que, atualmente, são identificados com o objeto matemático abstrato expressando um reconhecimento de realidade e aplicação prática na origem do desenvolvimento humano das Ciências.

O conhecimento construído a partir da colaboração mútua entre cientistas e cidadãos agrega valor tanto para a academia quanto para a sociedade. Projetos fundamentados nos princípios de Ciência Cidadã incentivam a participação das pessoas, de forma consciente e voluntária, não apenas na produção científica, mas também na resolução de problemas da sociedade.

\subsubsection{Projetos de Ciência Cidadã}

Atualmente, observa-se a aplicação de diversos projetos que se fundamentam na Ciência Cidadã, perpassando várias áreas de atuação científica. Muitos exemplos que se utilizam dessa abordagem podem ser citados. Um dos mais bem-sucedidos é o projeto $e B i r d$, um programa de 
listas on-line e em tempo real que tem revolucionado a forma como a comunidade de observadores de aves reporta e acessa informações sobre essas espécies de animais. Lançado no ano de 2002, pelo Cornell Lab of Ornithology e pela National Audubon Society, o eBird providencia dados com informações básicas sobre a abundância de aves e sua distribuição em variadas escalas espaciais e temporais (BONNEY et al., 2009).

Outras iniciativas de Ciência Cidadã são os projetos The Air Quality Egg (AQE), Asteroid Zoo e Cooperative Observer Program. O AQE foi projetado nos Estados Unidos da América (EUA) para possibilitar o monitoramento de poluentes no ar. O sistema permite a coleta de amostras do ar com instrumentos para a leitura das concentrações de poluentes em ambientes internos e externos (CITIZEN SENSE, 2013).

O Asteroid Zoo, executado pelo Zooniverse e pela Planetary Resources, visa à participação de voluntários na observação e classificação de asteroides desconhecidos localizados próximos à Terra (GREENEMEIER, 2014). Já o Cooperative Observer Program (COOP) é um projeto administrado pelo Serviço Nacional de Meteorologia dos EUA e pelos Centros Nacionais de Informações, que atua como uma rede cidadã composta por milhares de voluntários, que monitoram as condições climáticas nos 50 estados americanos (NATIONAL WEATHER SERVICE UNITED STATES DEPARTMENT OF COMMERCE, 2020).

No Brasil, a Ciência Cidadã ainda se encontra em estágio incipiente. Todavia, é possível identificar estudos em andamento. Alguns desses projetos são apresentados no Quadro 1.

\begin{tabular}{|l|l|}
\hline \multicolumn{1}{|c|}{ PROJETO } & \multicolumn{1}{|c|}{ DESCRIÇÃO/OBJETIVOS } \\
\hline $\begin{array}{l}\text { Projeto Cidadão } \\
\text { Cientista }\end{array}$ & $\begin{array}{l}\text { Criado em 2014, pela Save Brasil, objetiva monitorar aves em Unidades } \\
\text { de Conservação parques urbanos nos estados da Bahia, Rio de Janeiro, } \\
\text { São Paulo e Paraná. As espécies observadas durante os monitoramentos } \\
\text { são inseridas na plataforma eBird (SAVE BRASIL, 2020). }\end{array}$ \\
\hline Brydes do Brasil & $\begin{array}{l}\text { Voltado a criar uma base de dados sobre as baleias-de-bryde } \\
\text { identificadas a partir de fotos da espécie em águas brasileiras. Visa a } \\
\text { ampliar o conhecimento sobre as baleias-de-bryde na Região Sudeste. } \\
\text { Também é usado como fonte de informações para a formulação de } \\
\text { políticas públicas de conservação e proteção de áreas marinhas } \\
\text { (BRYDES DO BRASIL, 2020; SISTEMA DE INFORMAÇÃo SOBRE } \\
\text { A BIODIVERSIDADE, 2020). }\end{array}$ \\
\hline $\begin{array}{l}\text { Exoss Citizen Science } \\
\text { Project }\end{array}$ & $\begin{array}{l}\text { Registra meteoros e desenvolve estudos astrométricos e astrofísicos. } \\
\text { Cataloga novos radiantes do hemisfério sul. Colabora com instituiçães } \\
\text { de ensino e pesquisas, contribuindo com a sociedade. E incentiva a } \\
\text { produção de artigos científicos (EXOSS CITIZEN SCIENCE } \\
\text { PROJECT, 2020). }\end{array}$ \\
\hline AeTrapp & $\begin{array}{l}\text { Possibilita o engajamento de comunidades no monitoramento de } \\
\text { populações de mosquitos Aedes, vetores de zika, dengue, chikungunya e } \\
\text { febre amarela urbana (AETRAPP, 2020). }\end{array}$ \\
\hline $\begin{array}{l}\text { Ecoa - Ecologia e } \\
\text { Ação }\end{array}$ & $\begin{array}{l}\text { Organização não governamental surgida em 1989, em Campo } \\
\text { Grande/MS, é formada por um grupo de pesquisadores que atuam em }\end{array}$ \\
\hline
\end{tabular}




\begin{tabular}{|l|l|}
\hline \multicolumn{1}{|c|}{ PROJETO } & \multicolumn{1}{|c|}{ DESCRIÇÃO/OBJETIVOS } \\
\hline & $\begin{array}{l}\text { diversos segmentos profissionais, como: biologia; comunicação; } \\
\text { arquitetura; Ciências Sociais; engenharia; e educação. Seu principal } \\
\text { objetivo é estabelecer um espaço para reflexão, formulações, debates e } \\
\text { desenvolvimento de projetos epolíticas públicaspara aconservação } \\
\text { ambientale sustentabilidade (ECOA, 2020). }\end{array}$ \\
\hline $\begin{array}{l}\text { Universidade Federal } \\
\text { do ABC (UFABC) }\end{array}$ & $\begin{array}{l}\text { Desenvolve projetos de extensão que inclui estudos sobre os benefícios } \\
\text { das árvores para a população; Bentos de costão rochoso; a existênciae } \\
\text { abundância de Euglenas em praias do litoral paulista; Pellets plásticos } \\
\text { nas praias; a percepção das crianças com relação aos resíduos sólidos } \\
\text { urbanos (LOPES, 2020). }\end{array}$ \\
\hline DemarCafé & $\begin{array}{l}\text { Trata-se de um aplicativo desenvolvido por pesquisadores no Sul de } \\
\text { Minas Gerais, no ano de 2017, que tem o propósito de auxiliar as equipes } \\
\text { de geoprocessamento na conferência de campo dos mapas de café } \\
\text { gerados por geotecnologias. Permite o refinamento de áreas de café, } \\
\text { minimizando os problemas decorrentes da classificação incorreta em } \\
\text { mapeamentos do agronegócio cafeeiro (CASTRO et al., 2017). }\end{array}$ \\
\hline $\begin{array}{l}\text { Explicabilidade de } \\
\text { software }\end{array}$ & $\begin{array}{l}\text { Projeto desenvolvido por pesquisadores da Pontifícia Universidade } \\
\text { Católica de Minas Gerais (PUC-Minas). Analisa como produzir um } \\
\text { software que tenha a capacidade de se auto explicar às pessoas e ao } \\
\text { mesmo tempo identificar o que as pessoas esperam desses softwares para } \\
\text { que sejam efetivos (PONCIANO, 2019). }\end{array}$ \\
\hline $\begin{array}{l}\text { Pensamento } \\
\text { computacional }\end{array}$ & $\begin{array}{l}\text { Desenvolvido pela PUC-Minas, utiliza o algoritmo em atividades } \\
\text { cotidianas das pessoas e/ou organizações, como programar viagem; } \\
\text { adquirir produtos; ou organizar tarefas (PONCIANO, 2019). }\end{array}$ \\
\hline
\end{tabular}

Quadro 1 - Projetos de Ciência Cidadã no Brasil

Fonte: Autores (2020).

Além de contribuir em investigações científicas, projetos fundamentados na Ciência Cidadã impulsionam o senso crítico coletivo e servem de instrumento na formulação e aplicação de políticas públicas. Diante do valor científico e social da abordagem, a Ciência Cidadã pode ser considerada importante ferramenta na Educação, ao contribuir para a aprendizagem significativa dos voluntários envolvidos nos projetos.

\section{METODOLOGIA}

Uma vez que o objetivo deste artigo é identificar quais são os objetos de estudo das principais pesquisas sobre Ciência Cidadã, foi realizada uma revisão bibliográfica sobre o tema. A metodologia aplicada possui abordagem qualitativa e caráter exploratório. De acordo com Gil (2002), a pesquisa qualitativa exploratória está voltada a descobrir ideias e soluções, na tentativa de adquirir familiaridade com o problema estudado. Dessa forma, a pesquisa é exploratória porque se busca conhecer quais são os objetos de estudo das principais publicações sobre Ciência Cidadã. A pesquisa também possui caráter descritivo porque apresenta, de forma detalhada, as características desses objetos de estudo (GIL, 2002). 
No primeiro momento, foi realizada uma busca generalizada com o termo "science" no título do trabalho, apenas para se obter uma dimensão da relevância do tema Ciência na literatura, o que resultou em 234.606 publicações, depois filtradas para 21.807 resultados, nos últimos cinco anos.

Para refinar a pesquisa e alcançar o objetivo proposto, foi realizada uma busca de publicações relacionadas ao tema Ciência Cidadã na base de dados internacional Web of Science, utilizando, como critério, o termo "Citizen Science" no título da publicação (dessa forma: TI= ("CITIZEN SCIENCE") e, foram obtidos 1.308 resultados. Devido à quantidade elevada de ocorrências, delimitou-se somente artigos publicados nos últimos cinco anos (2016 a 2020) e, assim, foram retornados 930 resultados.

Para reduzir a quantidade de resultados e as principais publicações sobre o tema da Ciência Cidadã, optou-se por delimitar artigos nos últimos cinco anos e que registrassem, no mínimo, 40 citações, o que resultou em 31 artigos científicos. Portanto, para este estudo, os 31 resultados, são, posteriormente, apresentados na análise dos dados no quadro 2 que organiza um resumo dos artigos internacionais. É importante ressaltar que a busca foi realizada até 29 de dezembro de 2020.

Os principais pontos focais desses artigos internacionais são pesquisas sobre biologia, conservação e ecologia (KOBORI et al., 2016; MAISTRELLO et al., 2016; SWANSON et al., 2016; BALLARD et al., 2017; CHANDLER et al., 2017; McKINLEYet al., 2017). Outros artigos são relacionados às Ciências Sociais e epidemiologia, estimulando a participação do público em questões ambientais e de saúde (CAPPA et al., 2016; LAND-ZANDSTRA et al., 2016; WOOLLEY et al., 2016; JERRET et al., 2017; PALMER et al., 2017). Há também autores que avaliam a qualidade dos dados coletados pelos cientistas cidadãos (KOSMALA et al., 2016).

Na próxima seção são apresentados e analisados os objetos de estudo dos 31 artigos mais citados na literatura, extraídos da Web of Science.

\section{RESULTADOS E DISCUSSÃO}

Ao analisar os objetos de estudo dos 31 artigos resultantes da pesquisa na base de dados Web of Science, percebe-se que a maior parte das publicações remete às aplicações e contribuições da Ciência Cidadã. Um segundo conjunto de publicações está relacionado aos conceitos teóricos da Ciência Cidadã. E os demais estudos dizem respeito aos fatores que motivam os cidadãos a participarem dos projetos de Ciência Cidadã; às implicações éticas e 
sociais no recrutamento de voluntários; e à qualidade dos dados coletados pelos voluntários envolvidos nos projetos de Ciência Cidadã.

O trabalho que possui o maior número de citações (211) é o artigo Citizen science can improve conservation science, natural resource management, and environmental protection e, trata das formas de utilização da Ciência Cidadã e o custo para implantação de um sistema de Ciência Cidadã em uma universidade. Os autores realizaram um levantamento dos últimos 10 anos de como a Ciência Cidadã é utilizada (McKINLEYet al., 2017).

Dentre os artigos mais citados, a maior parte trata das aplicações e potencialidades do uso da Ciência Cidadã em áreas, como biologia, geologia, meio ambiente, saúde, ecologia e conservação. Esses estudos demonstram que a Ciência Cidadã pode ser aplicada por meio de uma diversidade de projetos e para diversas questões, como o monitoramento da biodiversidade (AMANO; LAMMING; SUTHERLAND, 2016; CHANDLER et al., 2017; JOHNSTON et al., 2018); a ecologia e conservação nos diversos países (KOBORI et al., 2016; BURGESS et al., 2017); na informação geográfica de crowdsourcing (SEE et al., 2016); na análise de microbiomas humanos (McDONALD et al., 2018); e na classificação das espécies na vida selvagem (SWANSON et al., 2016); e de aves (KAMP et al., 2016).

Outras aplicações da Ciência Cidadã abrangem projetos para documentar quantitativamente os fluxos de inundações em bacias hidrográficas (LE COZ et al., 2016; ABBOTT et al., 2018); para categorizar imagens de representações de falhas no tempo em classes morfológicas (ZEVIN et al., 2017); e para colher informações a respeito de pragas invasoras de culturas agrícolas, como é demonstrado no estudo de Maistrello et al. (2016) sobre o halyomorpha halys, um percevejo encontrado na Ásia.

Algumas das publicações estão relacionadas ao uso da Ciência Cidadã para auxiliar na redução do viés espacial; no esforço de obter amostragem e melhorar a utilização de esquemas de registro de espécies (GELDMAN et al., 2016); para avaliar a viabilidade da população de borboletas-monarca no oeste da América do Norte (SCHULTZ et al., 2017); e na pesquisa de lente do Space Warps (MORE et al., 2016).

Iniciativas de Ciência Cidadã possibilitam a participação de não cientista, o que proporciona conhecimento aos participantes e, ao mesmo tempo, soma esforços à academia, às instituições e ao governo, para solucionar problemas nas comunidades (BROEDER et al., 2018). Dentre os artigos selecionados, a pesquisa de Palmer et al. (2017) sobre a transmissão de doença pelo mosquito tigre asiático (Aedes albopictus) na Espanha, ressalta a relevância da participação pública na ciência e considera que a Ciência Cidadã está posicionada para revolucionar a vigilância de doenças transmitidas por mosquitos em todo o mundo. A Ciência 
Cidadã, portanto, pode ser utilizada como ferramenta confiável para rastrear agentes transmissores de doenças epidemiológicas.

Jerret et al. (2017) estudaram a utilização dos sensores de poluição do ar para melhorar as estimativas de exposição para análises epidemiológicas e Ciência Cidadã. O estudo avalia a validade de um sensor pessoal de poluição do ar de baixo custo.

Os principais estudos sobre Ciência Cidadã mostram que projetos fundamentados nos princípios da Ciência Cidadã também podem ser utilizados no processo de ensino e aprendizagem, na área de Educação Ambiental, proporcionando aprendizagem significativa dos participantes (ABBOTT et al., 2018).

Um exemplo é o artigo de Ballard et al. (2017), intitulado Youth-focused citizen science: examining the role of environmental science learning and agency for conservation, que trata de programas de Ciência Cidadã voltados para a participação dos jovens emações de conservação. Os autores realizaram estudos de caso focados na juventude para analisar os processos e resultados de aprendizagem e atividades de administração para jovens, bem como contribuições para o gerenciamento de locais e espécies. Dois programas foram examinados: um sobre o monitoramento costeiro e outro sobre a qualidade da água, em vários locais, na área da baía de San Francisco, Califórnia (BALLARD et al., 2017).

No âmbito da educação e conservação, o artigo denominado Contributions to conservation out comes by natural history museum-led citizen science: examining evidence and next steps aponta as contribuições da Ciência Cidadã dos museus de história natural para a conservação. Os museus de história natural são cenários óbvios para conectar a Ciência e a educação em conservação, por meio da Ciência Cidadã (BALLARD et al., 2017).

O periódico Science of the Total Environment publicou o artigo Marine anthropogenic litter on British beaches: A 10-year nation wide assessment using citizen science data, que também trata da educação e conscientização relativas ao meio ambiente. A pesquisa aborda a importância dos projetos de Ciência Cidadã no intuito de aumentar a conscientização sobre questões ambientais e como potencialidade de levar a mudanças positivas em comportamentos e atitudes das pessoas. Analisa os dados coletados durante uma década (2005-2014) por voluntários da Marine Conservation Society (MCS), durante pesquisas de praia realizadas ao longo da costa britânica, com o objetivo de aumentar o conhecimento sobre a composição, distribuição espacial e tendências temporais de detritos costeiros (NELMS et al., 2017).

$\mathrm{Na}$ literatura, há alguns autores que se dedicaram ao estudo dos conceitos relacionados à Ciência Cidadã, como Kullenberg e Kasperowski (2016), responsáveis por uma descrição cientométrica do que o conceito de Ciência Cidadã implica. 
Bonney et al. (2016) realizaram um estudo teórico das quatro categorias compostas nos projetos de Ciência Cidadã: coleta de dados, processamento de dados, currículo e ciências comunitárias. E outros estudiosos apresentam o panorama da Ciência Cidadã em ecologia e meio ambiente e suas mudanças ao longo do tempo (POCOCK et al., 2017).

A motivação dos participantes, nos projetos de Ciência Cidadã, foi objeto de estudo de quatro dos artigos avaliados. O primeiro refere-se à motivação dos voluntários e os impactos percebidos na aprendizagem dos voluntários na participação no projeto holandês iSPEX, que está fundamentado nos princípios da Ciência Cidadã, em que grande número de pessoas transformou seus smartphones em dispositivos para medir o impactodos aerossóis na saúde e no meio ambiente (LAND-ZANDSTRA et al., 2016).

O segundo artigo explana sobre as motivações dos leigos para atuarem como voluntários no Great Pollinator Project, um projeto de Ciência Cidadã relacionado à conservação na cidade de Nova York (DOMROESE; JOHNSON, 2017). E o terceiro trata da interação face a face dos participantes com os pesquisadores e cientistas, considerada uma estratégia motivacional para a participação das pessoas em projetos de Ciência Cidadã (CAPPA et al., 2016).

O quarto artigo aborda as implicações éticas e sociais no recrutamento de voluntários para as práticas de Ciência Cidadã no contexto da biomedicina. O argumento central é a discussão do engajamento de não cientistas, analisando motivações e discorrendo sobre as maneiras desse envolvimento. Ponderam, os autores, tratar-se de um dever cívico, dada a própria natureza da Ciência, cujas descobertas e o desenvolvimento são um patrimônio público, ainda que sujeitos ao resguardo da propriedade intelectual, no que concerne, principalmente, ao seu uso tecnológico e inventivo (WOOLLEY et al., 2016).

A qualidade dos dados produzidos pelos participantes voluntários nos projetos de Ciência Cidadã foi objeto de estudo do artigo Assessing data quality in Citizen Science. Os resultados demonstram que diversos tipos de projetos de Ciência Cidadã podem produzir dados com precisão igual ou superior à dos profissionais (KOSMALA et al., 2016).

A seguir, é apresentado, no Quadro 2, o resumo dos 31 principais estudos internacionais sobre o tema da Ciência Cidadã, organizado porordem decrescente do número de citações.

\begin{tabular}{|c|l|c|c|l|}
\hline Título do Artigo & Autor(es) & Ano & $\begin{array}{c}\text { Número } \\
\text { de } \\
\text { Citações }\end{array}$ & Objeto de Estudo \\
\hline $\begin{array}{l}\text { Citizen science can improve } \\
\text { conservation science, natural } \\
\text { resource management, and } \\
\text { environmental protection }\end{array}$ & $\begin{array}{l}\text { McKINLEY, } \\
\text { D. Ct al. }\end{array}$ & 2017 & 211 & $\begin{array}{l}\text { As formas de utilização da Ciência } \\
\text { Cidadã e o custo para implantação de } \\
\text { um sistema de Ciência Cidadã em uma } \\
\text { universidade. }\end{array}$ \\
\hline
\end{tabular}




\begin{tabular}{|c|c|c|c|c|}
\hline Título do Artigo & Autor(es) & Ano & $\begin{array}{l}\text { Número } \\
\text { de } \\
\text { Citações }\end{array}$ & Objeto de Estudo \\
\hline $\begin{array}{l}\text { Crowdsourcing, Citizen } \\
\text { Science or Volunteered } \\
\text { Geographic Information? The } \\
\text { Current State of Crowdsourced } \\
\text { Geographic Information }\end{array}$ & SEE, L. et al. & 2016 & 144 & $\begin{array}{lcc}\text { O uso da Ciência Cidadã } & \text { na } \\
\text { informação } & \text { geográfica } & \text { de } \\
\text { crowdsourcing. } & & \end{array}$ \\
\hline $\begin{array}{l}\text { Assessing data quality in citizen } \\
\text { science }\end{array}$ & $\begin{array}{l}\text { KOSMALA, } \\
\text { M. et al. }\end{array}$ & 2016 & 139 & $\begin{array}{l}\text { A qualidade dos dados produzidos } \\
\text { pelos cidadãos cientistas voluntários } \\
\text { nos projetos de Ciência Cidadã. }\end{array}$ \\
\hline $\begin{array}{l}\text { Can citizen science enhance } \\
\text { public understanding of } \\
\text { science? }\end{array}$ & $\begin{array}{l}\text { BONNEY, R. } \\
\text { et al. }\end{array}$ & 2016 & 138 & $\begin{array}{l}\text { A documentação dos resultados } \\
\text { científicos da Ciência Cidadã. }\end{array}$ \\
\hline $\begin{array}{l}\text { What Is Citizen Science? - A } \\
\text { Scientometric Meta-Analysis }\end{array}$ & $\begin{array}{l}\text { KULLENBER, } \\
\text { G. C.; } \\
\text { KASPEROWS } \\
\text { KI, D. }\end{array}$ & 2016 & 136 & $\begin{array}{l}\text { Descrição cientométrica do que o } \\
\text { conceito de Ciência Cidadã implica. }\end{array}$ \\
\hline $\begin{array}{l}\text { Contribution of citizen science } \\
\text { towards international } \\
\text { biodiversity monitoring }\end{array}$ & $\begin{array}{l}\text { CHANDLER, } \\
\text { M. et al. }\end{array}$ & 2017 & 126 & $\begin{array}{l}\text { As contribuições da Ciência Cidadã } \\
\text { para monitoramento da biodiversidade. }\end{array}$ \\
\hline $\begin{array}{l}\text { American Gut: An Open } \\
\text { Platform for Citizen Science } \\
\text { Microbiome Research }\end{array}$ & $\begin{array}{l}\text { McDONALD, } \\
\text { D. et al. }\end{array}$ & 2018 & 117 & $\begin{array}{l}\text { A utilização da Ciência Cidadã para } \\
\text { análise de microbiomas de fezes } \\
\text { humanos em comparação com } \\
\text { amostras ambientais. }\end{array}$ \\
\hline $\begin{array}{l}\text { Citizen science: A new } \\
\text { approach to advance ecology, } \\
\text { education, and conservation }\end{array}$ & $\begin{array}{l}\text { KOBORI, H. et } \\
\text { al. }\end{array}$ & 2016 & 96 & $\begin{array}{l}\text { O uso da Ciência Cidadã em questões- } \\
\text { chave relacionadas à ecologia e } \\
\text { conservação. }\end{array}$ \\
\hline $\begin{array}{l}\text { Marine anthropogenic litter on } \\
\text { British beaches: A 10-year } \\
\text { nationwide assessment using } \\
\text { citizen science data }\end{array}$ & $\begin{array}{l}\text { NELMS, S. E. } \\
\text { et al. }\end{array}$ & 2017 & 95 & $\begin{array}{l}\text { A importância dos projetos de Ciência } \\
\text { Cidadã no intuito de aumentar a } \\
\text { conscientização sobre questões } \\
\text { ambientais e como potencialidade de } \\
\text { levar a mudanças positivas em } \\
\begin{array}{l}\text { comportamentos e atitudes das } \\
\text { pessoas. }\end{array}\end{array}$ \\
\hline $\begin{array}{l}\text { A generalized approach for } \\
\text { producing, quantifying, and } \\
\text { validating citizen science data } \\
\text { from wildlife images }\end{array}$ & $\begin{array}{l}\text { SWANSON, } \\
\text { A. et al. }\end{array}$ & 2016 & 81 & $\begin{array}{l}\text { As classificações de espécies baseadas } \\
\text { no voto da pluralidade de cientistas } \\
\text { cidadãos múltiplos para fornecer uma } \\
\text { base confiável de dados para o } \\
\text { monitoramento em larga escala da vida } \\
\text { selvagem africana. }\end{array}$ \\
\hline $\begin{array}{l}\text { The science of citizen science: } \\
\text { Exploring barriers to use as a } \\
\text { primary research tool }\end{array}$ & $\begin{array}{l}\text { BURGESS, H. } \\
\text { K. et al. }\end{array}$ & 2017 & 77 & $\begin{array}{l}\text { As barreiras ao uso da Ciência Cidadã } \\
\text { como ferramenta de pesquisa na } \\
\text { biodiversidade. }\end{array}$ \\
\hline $\begin{array}{l}\text { Crowdsourced data for flood } \\
\text { hydrology: Feedback from } \\
\text { recent citizen science projects in } \\
\text { Argentina, France and New } \\
\text { Zealand }\end{array}$ & $\begin{array}{l}\text { LE COZ, J. et } \\
a l .\end{array}$ & 2016 & 70 & $\begin{array}{l}\text { Os projetos de Ciência Cidadã } \\
\text { utilizados para documentar } \\
\text { quantitativamente os fluxos de } \\
\text { inundações em bacias hidrográficas e } \\
\text { áreas urbanas da Argentina, França e } \\
\text { Nova Zelândia. }\end{array}$ \\
\hline $\begin{array}{lr}\begin{array}{l}\text { Gravity Spy: } \\
\text { advanced }\end{array} & \begin{array}{r}\text { Integrating } \\
\text { Ligodetector }\end{array} \\
\text { characterization, } & \text { machine } \\
\text { learning, and citizen science }\end{array}$ & $\begin{array}{l}\text { ZEVIN, M. et } \\
\text { al. }\end{array}$ & 2017 & 66 & $\begin{array}{l}\text { Um método que combina o } \\
\text { crowdsourcing com o aprendizado de } \\
\text { máquina para ajudar na tarefa de } \\
\text { categorizar as falhas registradas pelos } \\
\text { detectores Ligo. }\end{array}$ \\
\hline $\begin{array}{l}\text { The diversity and evolution of } \\
\text { ecological and environmental } \\
\text { citizen science }\end{array}$ & $\begin{array}{l}\text { POCOCK, M. } \\
\text { J. et al. }\end{array}$ & 2017 & 60 & $\begin{array}{l}\text { O panorama da Ciência Cidadã em } \\
\text { ecologia e meio ambiente e suas } \\
\text { mudanças ao longo do tempo. }\end{array}$ \\
\hline
\end{tabular}




\begin{tabular}{|c|c|c|c|c|}
\hline Título do Artigo & Autor(es) & Ano & $\begin{array}{l}\text { Número } \\
\text { de } \\
\text { Citações }\end{array}$ & Objeto de Estudo \\
\hline $\begin{array}{l}\text { Citizen science or scientific } \\
\text { citizenship? Disentangling the } \\
\text { uses of public engagement } \\
\text { rhetoric in national research } \\
\text { initiatives }\end{array}$ & $\begin{array}{l}\text { WOOLLEY, J. } \\
\text { P. et al. }\end{array}$ & 2016 & 55 & $\begin{array}{l}\text { As implicações éticas e sociais no } \\
\text { recrutamento de cidadãos cientistas em } \\
\text { práticas de Ciência Cidadã no contexto } \\
\text { da biomedicina. }\end{array}$ \\
\hline $\begin{array}{l}\text { Youth-focused citizen science: } \\
\text { Examining the role of } \\
\text { environmental science learning } \\
\text { and agency for conservation }\end{array}$ & $\begin{array}{l}\text { BALLARD, H. } \\
\text { L.; DIXON, C. } \\
\text { G. H.; } \\
\text { HARRIS, E. } \\
\text { M. }\end{array}$ & 2017 & 55 & $\begin{array}{l}\text { Programas de Ciência Cidadã voltados } \\
\text { para a participação dos jovens nas } \\
\text { ações de conservação. }\end{array}$ \\
\hline $\begin{array}{l}\text { Citizen science on a } \\
\text { smartphone: Participants' } \\
\text { motivations and learning }\end{array}$ & $\begin{array}{l}\text { LAND- } \\
\text { ZANDSTRA et } \\
\text { al. }\end{array}$ & 2016 & 54 & $\begin{array}{l}\text { A motivação dos voluntários e os } \\
\text { impactos percebidos na aprendizagem } \\
\text { dos cidadãos cientistas na participação } \\
\text { no projeto holandês iSPEX. }\end{array}$ \\
\hline $\begin{array}{l}\text { Why watch bees? Motivations } \\
\text { of citizen science volunteers in } \\
\text { the Great Pollinator Project }\end{array}$ & $\begin{array}{l}\text { DOMROESE, } \\
\text { M. C.; } \\
\text { JOHNSON, E. } \\
\text { A. }\end{array}$ & 2017 & 53 & $\begin{array}{l}\text { As motivações dos cientistas cidadãos } \\
\text { para atuarem como voluntários no } \\
\text { Great Pollinator Project. }\end{array}$ \\
\hline $\begin{array}{l}\text { Citizen science and early } \\
\text { detection of invasive species: } \\
\text { Phenology of first occurrences } \\
\text { of Halyomorphahalys in } \\
\text { Southern Europe }\end{array}$ & $\begin{array}{l}\text { MAISTRELLO } \\
\text { L. et al. }\end{array}$ & 2016 & 52 & 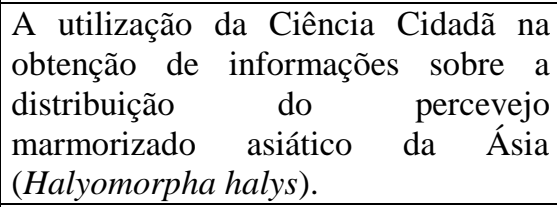 \\
\hline $\begin{array}{l}\text { Spatial gaps in global } \\
\text { biodiversity information and the } \\
\text { role of citizen science }\end{array}$ & $\begin{array}{l}\text { AMANO, T.; } \\
\text { LAMMING, J. } \\
\text { D. L.; } \\
\text { SUTHERLAN } \\
\text { D, W. J. }\end{array}$ & 2016 & 50 & $\begin{array}{l}\text { As potencialidades e limitações dos } \\
\text { projetos de Ciência Cidadã para } \\
\text { abordar lacunas nas informações sobre } \\
\text { biodiversidade, sobretudo da } \\
\text { perspectiva da conservação da } \\
\text { biodiversidade. }\end{array}$ \\
\hline $\begin{array}{l}\text { Validating novel air pollution } \\
\text { sensors to improve exposure } \\
\text { estimates for epidemiological } \\
\text { analyses and citizen science }\end{array}$ & $\begin{array}{l}\text { JERRET, M. et } \\
\text { al. }\end{array}$ & 2017 & 46 & $\begin{array}{l}\text { A validade de um sensor pessoal de } \\
\text { poluição do ar de baixo custo. }\end{array}$ \\
\hline 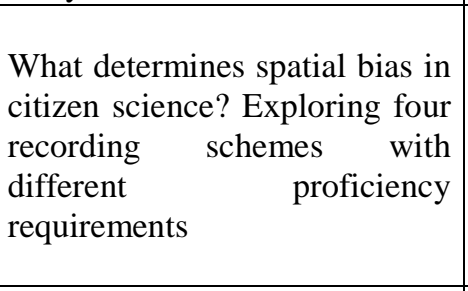 & $\begin{array}{l}\text { GELDMAN, J. } \\
\text { et al. }\end{array}$ & 2016 & 46 & $\begin{array}{l}\text { A integração de dados espaciais } \\
\text { contextuais na cobertura da terra e na } \\
\text { infraestrutura humana pode ajudar a } \\
\text { reduzir o viés espacial no esforço de } \\
\text { amostragem e melhorar a utilização de } \\
\text { esquemas de registro de espécies } \\
\text { baseados na Ciência Cidadã. }\end{array}$ \\
\hline Citizen science for public health & $\begin{array}{l}\text { BROEDER, D. } \\
\text { et al. }\end{array}$ & 2018 & 45 & $\begin{array}{l}\text { O envolvimento dos cidadãos na } \\
\text { produção de conhecimento. }\end{array}$ \\
\hline $\begin{array}{l}\text { Trends and seasonality of river } \\
\text { nutrients in agricultural } \\
\text { catchments: } 18 \text { years of weekly } \\
\text { citizen science in France }\end{array}$ & $\begin{array}{l}\text { ABBOTT, B. } \\
\text { W. et al. }\end{array}$ & 2018 & 45 & $\begin{array}{l}\text { A Ciência Cidadã como iniciativa para } \\
\text { alunos do ensino médio coletarem } \\
\text { amostras de bacias hidrográficas na } \\
\text { França. }\end{array}$ \\
\hline $\begin{array}{l}\text { Contributions to conservation } \\
\text { outcomes by natural history } \\
\text { museum-led citizen science: } \\
\text { Examining evidence and next } \\
\text { steps }\end{array}$ & $\begin{array}{l}\text { BALLARD, H. } \\
\text { et al. }\end{array}$ & 2017 & 44 & $\begin{array}{l}\text { As contribuições da Ciência Cidadã } \\
\text { dos museus de história natural para a } \\
\text { conservação. }\end{array}$ \\
\hline $\begin{array}{l}\text { Activating social strategies: } \\
\text { Face-to-face interaction in } \\
\text { technology-mediated citizen } \\
\text { science }\end{array}$ & $\begin{array}{l}\text { CAPPA, F. et } \\
\text { al. }\end{array}$ & 2016 & 43 & $\begin{array}{l}\text { A interação cara a cara dos cidadãos } \\
\text { com os cientistas utilizada como } \\
\text { estratégia motivacional para a }\end{array}$ \\
\hline
\end{tabular}




\begin{tabular}{|c|c|c|c|c|}
\hline Título do Artigo & Autor(es) & Ano & $\begin{array}{l}\text { Número } \\
\text { de } \\
\text { Citações }\end{array}$ & Objeto de Estudo \\
\hline & & & & $\begin{array}{l}\text { participação dos cidadãos em projetos } \\
\text { de Ciência Cidadã. }\end{array}$ \\
\hline $\begin{array}{l}\text { Space Warps-II. New } \\
\text { gravitational lens candidates } \\
\text { from the CFHTLS discovered } \\
\text { through citizen science }\end{array}$ & $\begin{array}{l}\text { MORE, A. et } \\
\text { al. }\end{array}$ & 2016 & 41 & $\begin{array}{l}\text { A Ciência Cidadã utilizada na pesquisa } \\
\text { de lente do Space Warps. }\end{array}$ \\
\hline $\begin{array}{l}\text { Citizen science monitoring } \\
\text { demonstrates dramatic declines } \\
\text { of monarch butterflies in } \\
\text { western North America }\end{array}$ & $\begin{array}{l}\text { SCHULTZ, C. } \\
\text { B. et al. }\end{array}$ & 2017 & 41 & $\begin{array}{l}\text { A Ciência Cidadã utilizada como } \\
\text { abordagem para avaliar a viabilidade } \\
\text { da população de borboletas-monarca } \\
\text { no oeste da América do Norte. }\end{array}$ \\
\hline 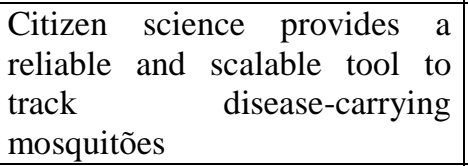 & $\begin{array}{l}\text { PALMER, J. R. } \\
\text { B. et al. }\end{array}$ & 2017 & 41 & $\begin{array}{l}\text { A Ciência Cidadã como ferramenta } \\
\text { confiável e escalável para rastrear o } \\
\text { mosquito tigre asiático (Aedes } \\
\text { albopictus) na Espanha. }\end{array}$ \\
\hline $\begin{array}{l}\text { Estimates of observer expertise } \\
\text { improve species distributions } \\
\text { from citizen science data }\end{array}$ & $\begin{array}{l}\text { JOHNSTON, } \\
\text { A. et al. }\end{array}$ & 2018 & 41 & $\begin{array}{l}\text { Monitoramento da ocupação de } \\
\text { espécies individuais. }\end{array}$ \\
\hline $\begin{array}{l}\text { Unstructured citizen science } \\
\text { data fail to detect long-term } \\
\text { population declines of common } \\
\text { birds in Denmark }\end{array}$ & KAMP, J. et al. & 2016 & 40 & $\begin{array}{l}\text { A correlação das tendências estimadas } \\
\text { a partir de dados não estruturados da } \\
\text { Ciência Cidadã. }\end{array}$ \\
\hline
\end{tabular}

Quadro 2 - Resumo dos principais estudos sobre Ciência Cidadã

Fonte: Web of Science (2020).

Os artigos analisados apontam que a Ciência Cidadã pode ser aplicada auma ampla variedade de objetos de estudo, nos diversos campos do conhecimento. Além disso, confirmam que projetos de Ciência Cidadã aumentam a conscientização sobre questões ambientais e podem produzir mudanças positivas em comportamentos e atitudes das pessoas.

A Ciência Cidadã aproxima os cidadãos de causas ambientais e sociais e aumenta a produção de conhecimento constituindo-se em formas singulares e criativas de envolvimento de não cientistas na construção do conhecimento, democratizando, dessa forma, tanto o acesso ao conhecimento como a sua produção.

\section{CONSIDERAÇÕES FINAIS}

O objetivo central que permeou este artigo esteve centrado em identificar quais são os objetos de estudo das principais pesquisas sobre Ciência Cidadã publicadas nos últimos cinco anos (2016-2020). Ao atingir o objetivo, constatou-se que a maior parte das publicações se refere às aplicações e contribuições da Ciência Cidadã. Os demais estudos tratam dos conceitos teóricos; dos fatores que motivam os cidadãos a participarem dos projetos de Ciência Cidadã; das implicações éticas e sociais no recrutamento de voluntários; e da qualidade dos dados coletados. 
Verificou-se, também, que a Ciência Cidadã propicia uma oportunidade de engajamento do público com a Ciência, com o método científico e com novas possibilidades educacionais que podem ser colocadas à disposição da sociedade. E, ainda, que o conhecimento científico pode ser construído com a participação do cidadão não cientista.

A despeito da relevância das pesquisas que envolvem a Ciência Cidadã e da grande quantidade de artigos publicados na área, ainda são poucos os estudos realizados no Brasil. Assim, o conteúdo deste artigo pode contribuir e motivar novas pesquisas na área.

Os docentes podemse utilizar da Ciência Cidadã para trabalhar os diversos componentes curriculares, no intuito de proporcionar uma aprendizagem significativa. Sugere-se que sejam implementados, na comunidade escolar, projetos de Ciência Cidadã que abordem a problemática da produção e do descarte do lixo, por se tratar de um dos desafios da humanidade atualmente.

A participação de docentes e alunos em projetos de Ciência Cidadã proporciona o engajamento em processos de pensamentos semelhantes aos que ocorrem em investigações científicase aumenta o seu conhecimento em áreas da ecologia, conservação, proteção ao meio ambiente e cidadania, entre outras.

Nesse sentido, para que a Ciência Cidadã chegue até as escolas e contribua para a formação científica dos alunos, os professores desempenham um papel central e precisam conhecer os procedimentos e protocolos derivados da Ciência Cidadã. Cursos de formação continuada proporcionados por ações de extensão universitária, por exemplo, podem contribuir para a interação entre professores da Educação Básica e pesquisadores, possibilitando reflexões sobre a Ciência Cidadã e outras maneiras de tornar o ensino de Ciências mais contextualizado e criativo.

\section{REFERÊNCIAS}

ABBOTT, B. W. et al. Trends and seasonality of river nutrients in agricultural catchments: 18 years of weekly citizen science in France. Science of the Total Environment, Amsterdam v. 624, p. 845-858, 2018.

AETRAPP. Monitoramento cidadão de focos de mosquitos Aedes, transmissores de dengue, zika, chikungunya e febre amarela urbana. Comunidades gerando dados para o combate aos mosquitos. Disponível em: https://www.aetrapp.org/. Acesso em: 27 mar. 2020.

AMANO, T.; LAMMING, J. D. L.; SUTHERLAND, W. J. Spatial gaps in global biodiversity information and the role of citizen science. BioScience, Oxford, v. 66, n. 5, p. 393-400, 2016.

AUSUBEL, D. The psychology of meaningful verbal learning. New York: Grune \& Stratton, 1963. 
BALLARD, H. L. et al. Contributions to conservation outcomes by natural history museumled citizen science: examining evidence and next steps. Biological Conservation, Essex, v. 208, p. 87-97, 2017.

BALLARD, H. L.; DIXON, C. G. H.; HARRIS, E. M. Youth-focused citizen science: examining the role of environmental science learning and agency for conservation. Biological Conservation, Essex, v. 208, p. 65-75, 2017.

BERTIN, P. R. B. a parceria para governo aberto como plataforma para o avanço da ciência aberta no Brasil. Transinformação, Campinas, v. 31, e190020, 2019.

BONNEY, R. Citizen science: a lab tradition. Living Bird, Nova York, v. 15, p. 7-15, 1996.

BONNEY, R. et al. Citizen science: a developing tool for expanding science knowledge and scientific literacy. BioScience, Oxford, v. 59, n. 1, p. 977-984, 2009.

BONNEY, R. et al. Can citizen science enhance public understanding of science? Public Understanding of Science, New York, v. 25, p. 2-16, 2016.

BROEDER, D. et al. Citizen science for public health. Health Promotion Internacional, London, v. 33, n. 3, p. 505-514, 2018.

BRYDES DO BRASIL. Programa de pesquisa participativa. Disponível em: http://www.brydesdobrasil.com.br/. Acesso em: 27 mar. 2020.

BURGESS, H. K. et al. The science of citizen science: exploring barriers to use as a primary research tool. Biological Conservation, Essex, v. 208, p. 113-120, 2017.

CAPPA, F.; LAUT, J.; NOV, O.; GIUSTINIANO, L.; PORFIRI, M. Activating social strategies: face-to-face interaction in technology-mediated citizen science. Journal of Environmental Management, London, v.182, p. 374-384, 2016.

CASTRO, Y. B. et al. Solução tecnológica para promover a ciência cidadã no mapeamento de áreas cafeeiras em Minas Gerais. In: SIMPÓSIO BRASILEIRO DE SENSORIAMENTO REMOTO, 2017, Santos. Anais [...]. Santos, 2017, p. 5636-5643.

CHANDLER, M. et al. Contribution of citizen science towards international biodiversity monitoring. Biological Conservation, Essex, v. 213, p. 280-294, 2017.

CITIZEN SENSE. Air Quality Egg and the Makers. 2013. Disponível em: https://citizensense.net/air-quality-egg/. Acesso em: 27 mar. 2020.

CLÍNIO, A. Ciência aberta na América Latina: duas perspectivas em disputa. Transinformação, Campinas, v. 31, 2019.

COHN, J. P. Citizen science: can volunteers do real research? BioScience, Oxford, v. 58, n. 3, p. 192-197, 2008.

D’AMBRÓSIO, U. Etnomatemática: elo entre as tradições e a modernidade. Belo Horizonte: Autêntica, 2001. 
DICKINSON, J.; ZUCKERBERG, B.; BONTER, D. Citizen science as anecological research tool. Annual Review of Ecology and Systematics, Palo Alto, v. 41, n. 1, p. 149-172, 2010.

DOMITE, M. C. S. Quando a etnomatemática entra em ação. Scientific American Brasil Etnomatemática, São Paulo, n. 11, p. 80-84, 2005.

DOMROESE, M. C.; JOHNSON, E. A. Why watch bees? motivations of citizen science volunteers in the great pollinator project. Biological Conservation, Essex, v. 208, p. 40-47, 2017.

ECOA. Disponível em: https://ecoa.org.br/ecoa-institucional/. Acesso em: 27 mar. 2020.

EGLASH, R. Fractais Africanos. Scientific American Brasil-Etnomatemática, São Paulo, n. 11, p. 66-67, 2005.

EXOSS CITIZEN SCIENCE PROJECT. Monitoramento de meteoros. Disponível em: http://press.exoss.org/projetos/. Acesso em: 27 mar. 2020.

GELDMAN, J. et al. What determines spatial bias in citizen science? exploring four recording schemes with different proficiency requirements. Diversity and Distributions, Cambridge, v. 22, p. 1139-1149, 2016.

GIL, A. C. Como elaborar projetos de pesquisa. 4. ed. São Paulo: Atlas, 2002.

GREENEMEIER, L. Scientists scanning our solar system need your help to find asteroids for the exploration of their mineral properties. Scientific American, 2014. Disponível em: https://www.scientificamerican.com/citizen-science/zooniverse-asteroid-zoo/. Acesso em: 27 mar. 2020.

HAYOOD, B. K.; PARRISH, J. K.; DOLLIVER, J. Place-based and data-rich citizen science as a precursor for conservation action. Conservation Biology, Nova York, v. 30, n. 3, p. 476486, 2016.

IRWIN, A. Citizen science: a study of people, expertise and sustainable development. London and NewYork: Routledge, 1995.

JASANOFF, S. Tecnologias da humildade participação cidadã na governança da ciência.

Sociedade e Estado, Brasília, v. 34, n. 2, 2019.

JERRETT, M. et al. Validating novel air pollution sensors to improve exposure estimates for epidemiological analyses and citizen science. Environmental Research, Elsevier, Amsterdam, v. 158, p. 286-294, 2017.

JOHNSTON, A. et al. Estimates of observer expertise improve species distributions from citizen science data. Methods in Ecology and Evolution, London, v. 9, n. 1, p. 88-97, 2018.

KAMP, J. et al. Unstructured citizen science data fail to detect long-term population declines of common birds in Denmark. Diversity and Distributions, Cambridge, v. 22, p. 1024-1035, 2016. 
KOBORI, H. et al. Citizen science: a new approach to advance ecology, education, and conservation. Ecological Research, Kyoto, v. 31, n. 1, p. 1-19, 2016.

KOSMALA, M. et al. Assessing data quality in citizen science. Frontiers in Ecology and the Environment, Whashington, DC, v. 14, n. 1, p. 551-560, 2016.

KULLENBERG, C.; KASPEROWSKI, D. What is citizen science? ascientometricmetaanalysis. PlosOne, San Francisco, v. 11, n. 1, 2016.

LAND-ZANDSTRA, A. M. et al. Citizen science on a smartphone: participants motivations and learning. Public Understanding of Science, New York, v. 25, n. 1, p. 45-60, 2016.

LE COZ, J. et al. Crowdsourced data for flood hydrology: feedback from recent citizen science projects in Argentina, France and New Zealand. Journal of Hydrology, Elsevier, Amsterdam, v. 541, p. 766-777, 2016.

LOPES, N. P. G. Ciência cidadã. Disponível em:

http://professor.ufabc.edu.br/ natalia.lopes/cienciacidada/. Acesso em: 27 mar. 2020.

MAISTRELLO, L. et al. Citizen science and early detection of invasive species: phenology of first occurrences of Halyomorphahalys in southerneurope. Biological Invasions, Berkeley, v. 18, n. 11, p. 3109-3116, 2016.

McDONALD, D. et al. American gut: an open platform for citizen science microbiome Research. mSystems, Rockville, v. 3, n. 3, p. 1-28, 2018.

MCKINLEY, D. C. et al. Citizen science can improve conservation science, natural resource management, and environmental protection. Biological Conservation, Essex v. 208, p. 15-28, 2017.

MORE, A. et al. SPACE WARPS-II. New gravitational lens candidates from the CFHTLS discovered through citizen science. Monthly Notices of the Royal Astronomical Society, Oxford, v. 455, n. 2, 2016.

NATIONAL WEATHER SERVICE UNITED STATES DEPARTMENT OF COMMERCE. Cooperative Observer Program (COOP). Disponível em:

https://www.weather.gov/coop/overview. Acesso em: 27 mar. 2020.

NELMS, S. E. et al. Marine anthropogenic litter on british beaches: 10-year nationwide assessment using citizen science data. Science of the Total Environment, Amsterdam v. 579, p. 1399-1409, 2017.

PALMA, D. A. Monitoramento de qualidade da água com o enfoque ciência cidadã: estudo de cem Brazilândia. 2016. 76 p. Monografia (Projeto Final) Universidade de Brasília, Brasília, 2016.

PALMER, J. R. B. et al. Citizen science provides a reliable and scalable tool to track diseasecarrying mosquitoes. Nature Communications, London, v. 8, n. 916, 2017.

POCOCK, M. J. et al. The diversity and evolution of ecological and environmental citizen science. PlosOne, San Francisco, v. 12, n. 4, p. e0172579, 2017. 
PONCIANO, L. Estímulo à curiosidade e envolvimento com a pesquisa científica: comunidade é chamada a participar de projetos científicos pela PUC-Minas. Estado de Minas Educação, 2019. Disponível em:

https://www.em.com.br/app/noticia/especiais/educacao/2019/07/29/internas_educacao,10732 08/envolvimento-com-pesquisa.shtml. Acesso em: 24 mar. 2020.

REBOUCAS, F. Ciência cidadã- Pensegreen. Gazeta Brazilian News, 2013. Disponível em: http://gazetanews.com/ciencia-cidada-pense-green/. Acesso em: 12 jun. 2020.

SAVE BRASIL. Sociedade para a conservação das aves do Brasil. Disponível em: http://savebrasil.org.br/cidadao-cientista-1. Acesso em: 27 mar. 2020.

SCHULTZ, C. B. et al. Citizen science monitoring demonstrates dramatic declines of monarch butterflies in western north America. Biological Conservation, Essex, v. 214, p.343-346, 2017.

SEE, L. et al. Crowdsourcing, citizen science or volunteered geographic information? the current state of crowdsourced geographic information. ISPRS International Journal of Geoinformation, Basel, v. 5, n. 5, 2016.

SISTEMA DE INFORMAÇÃO SOBRE A BIODIVERSIDADE BRASILEIRA. SIBBR. Disponível em:

https://www.sibbr.gov.br/cienciacidada/projetos.html. Acesso em: 23 mar. 2020.

SOARES, M. D.; SANTOS, R. D. C. Ciência cidadã: o envolvimento popular em atividades científicas. Revista Ciência Hoje, São Paulo, v. 47, p. 38-43, 2011.

SOCIENTIZE. Project Socientizeannounces white paper on citizen science at their final conference, 2014. Disponível em:https://translate.google.com/translate?hl=ptBR\&sl=en\&u=https://ec.europa.eu/digital-single-market/en/news/project-socientizeannounces-white-paper-citizen-science-their-final-conference\&prev=search. Acesso em: 13 jun. 2020.

STEWART, I. Mosaicos e origami. Scientific American Brasil-Etnomatemática, São Paulo, n. 11, p. 78-79, 2005.

SWANSON, A. et al. A generalized approach for producing, quantifying, and validating citizen science data from wildlife images. Conservation Biology, New York, 30, n. 3, p. 520$531,2016$.

WILDSCHUT, D. The need for citizen science in the transition to a sustainable peer-to-peersociety. Futures, San Francisco, v. 91, p. 46-52, 2017.

WOOLLEY, J. P. et al. Citizen science or scientific citizenship? disentangling the uses of public engagement rhetoric in national research initiatives. BMC Medical Ethics, London, v.17, n. 33, 2016.

ZEVIN, M. et al. Gravity Spy: Integrating advanced LIGO detector characterization, machine learning, and citizen science. Classical and Quantum Gravity, Cambridge, v.1, 2016. 


\section{DADOS DOS AUTORES:}

Nome: Diny Gabrielly de Miranda Martins

E-mail: dinygabrielly@ hotmail.com

Curriculum Lattes: http://lattes.cnpq.br/1044358241127422

Mestranda em Administração Pública pela Universidade Federal de Lavras, especialização em Gestão Estratégica de Pessoas pela Universidade Cruzeiro do Sul, especialização em Docência pelo Instituto Federal de Minas Gerais - Campus Arcos e graduação em Administração pelo Instituto Federal de Minas Gerais - Campus Formiga. Docente dos cursos técnicos em Administração e Recursos Humanos e do Ensino Médio da rede pública de ensino da Secretaria de Estado de Educação de Minas Gerais.

Nome: Eloisa Helena de Souza Cabral

E-mail: elocabral@uol.com.br

Curriculum Lattes: http://lattes.cnpq.br/4823917786094769

Doutorado em Ciências Sociais pela Pontifícia Universidade Católica de São Paulo, mestrado em Serviço Social pela mesma Instituição e graduação em Serviço Social pela Faculdade de Serviço Social de Araraquara. Professora permanente/colaboradora do Mestrado Profissional em Administração Pública da Universidade Federal de Lavras. Professora convidada/colaboradora do Mestrado Profissional em Administração Pública da Universidade Federal dos Vales de Jequitinhonha e Mucuri. Professora do Centro de Estudos de Pessoal do Departamento de Educação do Exército Brasileiro. Desenvolve estudos e atividades na área do fomento à integração institucional da Universidade, Industria e Defesa Nacional. Realiza atividades de pesquisa nas áreas de: avaliação de impacto, econômica e ambiental, sociologia da cultura, sociologia econômica, responsabilidade social, desenvolvimento, sustentabilidade, gestão cultural, terceiro setor e gestão social. 